\title{
Solving boussinesq equation by bilinear bäcklund transformation
}

\author{
M. Y. Adamu* , E. Suleiman \\ Abubakar Tafawa Balewa University, Bauchi, Nigeria \\ Email address: \\ magajiadamu78@yahoo.com (M. Y. Adamu)
}

\section{To cite this article:}

M. Y. Adamu, E. Suleiman. Solving Boussinesq Equation by Bilinear Bäcklund Transformation, Applied and Computational Mathematics. Vol. 2, No. 2, 2013, pp. 32-35. doi: 10.11648/j.acm.20130202.13

\begin{abstract}
In this paper Hirota bilinear method is applied to constructing Backlund transformation of the Boussinesq equation. The bilimear Backlund form are used to obtain the soliton solution of the Boussinesq equation. Also as an application for the bilinear Bäcklund transformation, new classes of wave solutions to the Boussinesq Equation are computed.
\end{abstract}

Keywords: Boussinesq Equation, Backlund Transformation, Hirota Bilinear Form, Travelling Wave

\section{Introduction}

Searching for exact solution to nonlinear equation of mathematical physics are significantly important. The transformed rational function method [1] and multiple expfunction method [2] provide some generic approaches for constructing travelling wave solutions and multiple wave solutions respectively. Perturbation expansion method proposed by Hirota [3] often generates a specific class of multiple wave solution including the $\mathrm{N}$-soliton solutions, provided that the equation possesses a Hirota bilinear form. Also linear superposition principle may apply Hirota bilinear and in particular linear subspaces of solutions for nonlinear equations [4]. Another powerful approach to solution of nonlinear equation are the Bäcklund Transformations, they can be written as Hirota bilinear form when the equation under consideration has a bilinear form $[5,6]$. The Bilinear Bäcklund Transformation is essentially defined as pair of partial differential relation involving two independent variable and their derivatives, which together imply that each one of the independent variable satisfy a partial differential equation $[7,8]$. Thus, for example, the transformation

$$
\psi_{x}=F\left(\varphi, \psi, \varphi_{x}, x\right)
$$

would imply that $\varphi$ and $\psi$ satisfy partial differential equations of the operational form,

$$
P(\varphi)=0
$$

\section{Bilinear Form}

We consider the following (1+1)-dimensional nonlinear equation

$$
\frac{\partial^{2} u}{\partial t^{2}}+\frac{\partial^{2} u^{2}}{\partial x^{2}}+\frac{\partial^{4} u}{\partial x^{4}}=0
$$

Under the dependent variable transformation

$$
u=6 \frac{\partial}{\partial x}(\ln \tau)
$$

equation (1) can be mapped into the Hirota bilinear equation

$$
\left(D_{t}^{2}+D_{x}^{4}\right) \tau \cdot \tau=0
$$

where the bilinear differential operator D is defined by

$$
\begin{aligned}
& D_{t}^{m} D_{x}^{n} a(t, x) b(t, x)= \\
& \left.\frac{\partial^{m}}{\partial s^{m}} \frac{\partial^{n}}{\partial y^{n}} a(t+s, x+y) b(t-s, x-y)\right|_{s=0, y=0}
\end{aligned}
$$

We can re-write equation (1) in terms of $\tau$ as follows:

$$
\begin{aligned}
& \left(\frac{\partial^{4} \tau}{\partial x^{4}}+\frac{\partial^{2} \tau}{\partial t^{2}}\right) \tau-4 \frac{\partial^{3} \tau}{\partial x^{3}} \frac{\partial \tau}{\partial x} \\
& +3\left(\frac{\partial^{2} \tau}{\partial x^{2}}\right)^{2}-\frac{\partial \tau}{\partial t}=0
\end{aligned}
$$

In this work, we would like to present a bilinear Bä cklund transformation for equation (1) as proposed in [10]. 
Let us suppose that we have another solution $\tau^{\prime}$ to the equation

$$
\left(D_{t}^{2}+D_{x}^{4}\right) \tau^{\prime} \cdot \tau^{\prime}=0
$$

and we will produce the key function

$$
\mathrm{P}=\left[\left(D_{t}^{2}+D_{x}^{4}\right) \tau . \tau\right] \tau^{\prime 2}-\left[\left(D_{t}^{2}+D_{x}^{4}\right) \tau^{\prime} \cdot \tau^{\prime}\right] \tau^{2}(7)
$$

If $P=0$ then

$\tau$ is a solution of (3) $\Leftrightarrow \tau^{\prime}$ is a solution of (3)

Therefore, if we can obtain from $P=0$ by interchanging the dependant variable $\tau$ and $\tau^{\prime}$, a system of bilinear equation that guarantees $\mathrm{P}=0$

$$
\mathrm{B}_{i}\left(D_{t}, D_{x}\right) \tau \cdot \tau^{\prime}=0,1 \leq \mathrm{i} \leq \mathrm{M}
$$

where the $B_{i}$ 's are polynomial in the indicated variables and $\mathrm{M}$ is natural number depending on the complexity of the equation. A question now arises; is $P=0$ has unique solution? Otherwise, how to get all the solutions?

The answer to the first part of the equation may be No. for the second part of the equation, it is known that Hirota's bilinear operator identities are the key to break $\mathrm{P}$ into a system of polynomials $B_{i}$ 's the following the lemmas are useful in generating all the nontrivial identities. Lemma: Let $D=\mathbb{R}\left[f^{(i)}, g^{(j)}\right],(i, j \in J)$ be the polynomial algebra, where $\mathrm{J}$ denotes the space of multi-indices $\left(\left(i_{1}, i_{2}, . ., i_{n}\right)\right)$. In this algebra, we have the derivations $\partial_{m}(m=1, \ldots, n)$ Which act in the very direct way,

$$
\begin{aligned}
& \partial_{m}\left(f^{(i)}\right)=f^{\left(i+1_{m}\right)} \text { and } \partial_{m}\left(g^{(i)}\right)=g^{\left(j+1_{m}\right)} \\
& \text { where } 1_{m}=(0, \ldots, 0,1,0, \ldots, 0),
\end{aligned}
$$

Where $1_{m}=(0, \ldots, o, 1,0, \ldots, 0)$, the $m^{\text {th }}$ basis vector in the n-dimensional Euclidean space.

Lemma. Let $D_{m}: D \otimes D \rightarrow D \otimes D$ be the linear map:

$$
D_{m}(a \otimes b)=\partial_{m}(a) \otimes b-a \otimes \partial_{m}(b)
$$

Then

$$
\exp \left(D_{m}\right)(a \otimes b)=\exp \left(\partial_{m}\right) a \otimes \exp \left(-\partial_{m}\right)
$$

Observe that the image of the map $D_{m}$ is again in $D \otimes D$ and not in $D$ which makes this map not similar to the usual one. To get the usual Hirota derivatives [6] $D_{m}$ we have to project the image of $D_{m}$ to $D$ :

Letting $D \otimes D \rightarrow D, \Pi\left(\sum a_{i} \otimes b_{i}\right)=\sum a_{i} b_{i}$, be the projection map, then $D_{m}^{k}(a \otimes b)=\prod D_{m}^{k}(a \otimes b), \quad k=1,2,3, \ldots$.

Lemma: let $\partial_{\alpha}=\sum a_{i} \partial_{i}, \partial_{\beta}=\sum \beta_{i} \partial_{i}$ and $\partial_{\gamma}=\sum \gamma_{i} \partial_{i}$ and let $D_{\alpha}, D_{\beta}$ and $D_{\gamma}$ be the corresponding projected Hirota derivatives then we have all $a, b, c, d \in D$

$$
\begin{aligned}
& \exp \left(D_{\alpha}\right)\left[\exp \left(D_{p}\right)(a \otimes b) \otimes \exp \left(D_{\alpha}\right)(c \otimes d)\right]= \\
& \exp \left(\frac{1}{2}\left(D_{\beta}-D_{\alpha}\right)\right) \\
& {\left[\exp \left(\frac{1}{2}\left(D_{\beta}-D_{\alpha}\right)+D_{\alpha}\right)(a \otimes b)\right.} \\
& \left.\otimes \exp \left(\frac{1}{2}\left(D_{\beta}+D_{\alpha}\right)-D_{\alpha}\right)(a \otimes b)\right]
\end{aligned}
$$

Note that the above identity gives all possibility for remitting $P=0$ in the form

$$
\sum \beta_{i} \tau \cdot \tau^{\prime}=0
$$

through the relation $\exp \left(\sum \in_{i} D_{i}\right)=\prod \exp \left(\in_{i} D_{i}\right)$ for example, letting $k, l, m \in J$ by taking $a=b=\tau$ and $c=d=\tau$ the coefficient of $\alpha^{k} \beta^{l} \lambda^{m}$ in equation...(9) gives the following identity

$$
\begin{aligned}
& D^{k}\left[D^{l}(\tau \cdot \tau) \cdot D^{m}\left(\tau^{\prime} \cdot \tau^{\prime}\right)\right]= \\
& \sum D^{\cdots}\left[D^{\cdots}\left(\tau \cdot \tau^{\prime}\right) \cdot D^{\cdots}\left(\tau \cdot \tau^{\prime}\right)\right]
\end{aligned}
$$

it is easy to check that an expression of such type is not unique if we take $a=b=c=\tau$ and $c=d=\tau^{\prime}$

The coefficients of $\alpha^{k} \beta^{l} \lambda^{m}$ in the equation....(9) give the following identity

$$
\begin{aligned}
& D^{k}\left[D^{l}\left(\tau \cdot \tau^{\prime}\right) \cdot D^{m}\left(\tau \cdot \tau^{\prime}\right)\right]= \\
& \sum D^{\cdots}\left[D^{\cdots}\left(\tau \cdot \tau^{\prime}\right) \cdot D^{\cdots}\left(\tau \cdot \tau^{\prime}\right)\right]
\end{aligned}
$$

Let us now introduce the following identities which are useful for Hirota's bilinear operators:

$$
\begin{gathered}
D_{\eta}\left(D_{\zeta} a \cdot b\right) \cdot a b=D_{\zeta}\left(D_{\eta} a \cdot b\right) \cdot b a, \\
D_{\eta} a b \cdot c d=\left(D_{\eta} a \cdot b\right) c b-a d\left(D_{\eta} c \cdot b\right),(14) \\
b^{2}\left(D_{\zeta}^{2} a \cdot a\right)-\left(D_{\zeta}^{2} b \cdot b\right) a^{2}=2 D_{\zeta}\left(D_{\zeta} a \cdot b\right) \cdot b a,(15) \\
b^{2}\left(D_{\eta} D_{\zeta} a \cdot a\right)-\left(D_{\eta} D_{\zeta} b \cdot b\right) a^{2}=2 D_{\zeta}\left(D_{\eta} a \cdot b\right) \cdot b a \\
b^{2}\left(D_{\eta}^{3} D_{\zeta} a \cdot a\right)-\left(D_{\eta}^{3} D_{\zeta} b \cdot b\right) a^{2} \\
=2 D_{\zeta}\left(D_{\eta}^{3} a \cdot b\right) \cdot b a+6 D_{\eta}\left(D_{\eta} D_{\zeta} a \cdot b\right) \cdot\left(D_{\eta} b \cdot a\right)
\end{gathered}
$$

Note that the above identity can be obtained from the identity in lemma 7 for more identities and general exchange formulae, you are referred to see [9] Applying the above identities to equation (5) We can obtain

$$
\tau^{\prime 2}\left(D_{t} \tau . \tau\right)-\left(D_{t}^{2} \tau^{\prime} \cdot \tau^{\prime}\right) \tau^{2}=2 D_{t}\left(D_{t} \tau \cdot \tau^{\prime}\right) \cdot \tau^{\prime} \tau
$$

and

$$
\begin{aligned}
& \tau^{1^{2}}\left(D_{x}^{4} \tau \cdot \tau\right)-\left(D_{x}^{4} \tau^{1} \cdot \tau^{1}\right) \tau^{2}= \\
& 2 D_{x}\left(D_{x}^{3} \tau \cdot \tau^{1}\right) \cdot \tau^{1} \tau+6 D_{x}\left(D_{x}^{2} \tau \cdot \tau^{1}\right) .\left(D_{x} \tau^{1} \cdot \tau\right)
\end{aligned}
$$


Substituting the above result into the right hand side of equation (5), we can obtain

$$
\begin{aligned}
& P=2 D_{t}\left(D_{t} \tau \cdot \tau^{\prime}\right) \cdot \tau^{\prime} \tau+2 D_{x}\left(D_{x}^{3} \tau \cdot \tau^{\prime}\right) \cdot \tau^{\prime} \tau \\
& +6 D_{x}\left(D_{x}^{2} \tau \cdot \tau^{\prime}\right) .\left(D_{x} \tau^{\prime} \cdot \tau\right)
\end{aligned}
$$

One can observe that $\mathrm{P}=0$ has more than one unique solution, and the Hirota's bilinear operators identities of the type (13)-(17) was not able to provide general bilinear systems. We will then use to resulting transformation to obtain multiple solution to equation (1) let us now introduce new arbitrary parameters $\eta$ are $\square_{i}(\mathrm{i}=1,2,3)$ into equation (20) to obtain

$$
\begin{aligned}
& P_{ \pm}^{a}=2 D_{t}\left[\left(D_{t} \pm 3 \eta D_{x} \mp \varepsilon_{i}\right) \tau \cdot \tau^{1}\right] \cdot \tau^{1} \tau+ \\
& 2 D_{x}\left[\left(D_{x}^{3} \mp 3 \eta D_{t} \pm \varepsilon_{2}\right) \tau \cdot \tau^{1}\right] \tau^{1} \cdot \tau+ \\
& 6 D_{x}\left[\left(D_{x}^{2} \pm \eta D_{x}\right) \tau \cdot \tau^{1}\right] \cdot\left(D_{x} \tau^{1} \cdot \tau\right)
\end{aligned}
$$

this is possible because the coefficient of $\eta, \varepsilon_{1}$ and $\varepsilon_{2}$

$$
\begin{gathered}
\eta: \pm 6 D_{t}\left(D_{x} \tau \cdot \tau^{\prime}\right) \cdot \tau^{\prime} \tau \mp 6 D_{x}\left(D_{t} \tau \cdot \tau^{\prime}\right) \cdot \tau^{\prime} \tau \\
\varepsilon_{1}: \mp 2\left(D_{t} \tau \cdot \tau^{\prime}\right) \cdot \tau^{\prime} \tau \\
\varepsilon_{2}: \pm 2\left(D_{t} \tau \tau^{\prime}\right) \cdot \tau^{\prime} \tau \\
\mu: \pm 6 D_{x}\left[D_{x} \tau \cdot \tau^{\prime}\right] .\left(D_{x} \tau^{\prime} \cdot \tau\right)
\end{gathered}
$$

are all equal to zero due to the following properties

$$
\begin{gathered}
D_{\zeta} f \cdot f=0 \\
D_{\zeta} f \cdot g=-D_{\zeta} g \cdot f \\
D_{\eta}\left(D_{\zeta} f \cdot g\right) \cdot g f=D_{\zeta}\left(D_{\eta} f \cdot g\right) \cdot g f
\end{gathered}
$$

Then $p_{ \pm}^{a}=0$ if $B_{i} \tau \cdot \tau^{1}=0,1 \leq i \leq 3$ where $B_{i}{ }^{\prime} s$ can be found from equation (21) as follows

$$
\left\{\begin{array}{l}
B_{i} \tau . \tau^{\prime} \equiv\left(D_{t} \pm 3 \eta D_{x} \mp \varepsilon_{1}\right) \tau . \tau^{\prime} \\
B_{2} \tau \cdot \tau^{\prime} \equiv\left(D_{x}^{3} \mp 3 \eta D_{t} \pm \varepsilon_{2}\right) \tau . \tau^{\prime} \\
B_{3} \tau . \tau^{\prime} \equiv\left(D_{x}^{2} \pm \mu D_{x}\right) \tau . \tau^{\prime}
\end{array}\right.
$$

the coefficients of $\eta, \mu, \varepsilon_{1}$ and $\varepsilon_{2}$ are equal to zero because of the equation (22)-(24) this shows that the system (25) presents a bilinear bäcklund transformation for (1).

Travelling Wave Solution

We now present an application of the bilinear Bäcklund transformation (25) in order to construct a new class of solution to equation (1). For this reason, we begin with $\tau=1$, which is the trivial solution of the equation (3), obviously noting that

$$
D_{\eta}^{n} \varphi .1=\frac{\partial^{n} \varphi}{\partial \eta^{n}}, n \geq 1
$$

the bilinear Bäcklund transformation (25) associated with $\tau=1$ become a system of linear partial differential equations:

$$
\left\{\begin{array}{l}
\frac{\partial \tau^{\prime}}{\partial t} \pm 3 \eta \frac{\partial \tau^{\prime}}{\partial x} \mp \varepsilon_{1} \tau^{\prime}=0 \\
\frac{\partial^{3} \tau^{\prime}}{\partial x^{3}} \mp 3 \eta \frac{\partial \tau^{\prime}}{\partial t} \pm \varepsilon_{2} \tau^{\prime}=0 \\
\frac{\partial^{3} \tau^{\prime}}{\partial x^{3}} \mp 3 \eta \frac{\partial \tau^{\prime}}{\partial t} \pm \varepsilon_{2} \tau^{\prime}=0 \\
\frac{\partial^{2} \tau^{\prime}}{\partial x^{2}} \pm \mu \frac{\partial \tau^{\prime}}{\partial x}=0
\end{array}\right.
$$

Let us consider a class of exponential wave solution of the form

$$
\tau^{1}=1+\varepsilon e^{k x-w t+\zeta^{0}}, \zeta^{0}=\cos t
$$

where $\varepsilon, k$ and $w$ are constants to be determine. On selecting

$$
\varepsilon_{1}=0, \varepsilon_{2}=0
$$

and after some straightforward calculation, we can set

$$
w= \pm 3 \eta k, \mu=\mp k
$$

therefore, we obtain a class of exponential wave solution to the equation (3)

$$
\tau_{ \pm}^{1}=1+\varepsilon \exp \left(k x \mp 3 \eta k t+\zeta^{0}\right)
$$

where $\varepsilon, k, \eta$ and $\mu$ are arbitrary constants; and

$$
U=6\left(\operatorname{In} \tau_{ \pm}^{1}\right)_{x}
$$

solves equation (1).

Let us now consider a second class of first order polynomial solution

$$
\tau^{\prime}=k x-w t
$$

where $\mathrm{k}$ and $w$ are constants to determined again on selecting

$$
\varepsilon_{1}=0, \varepsilon_{2}=0
$$

a direct computation shows that the system (27) becomes

$$
\begin{gathered}
w= \pm 3 \eta k \\
\pm 3 \eta w=0 \\
\pm \mu k=0
\end{gathered}
$$

which gives $\tau_{ \pm}^{\prime}=0$

\section{Conclusions}


The bilinear Backlund Transformation for the nonlinear Boussinesq equation was constructed base on the existence of exchange identities for Hirota bilinear operators. Also and application for the bilinear Backlund transformation was used to construct a new class of exact wave solutions for the Boussinessq equation.

\section{References}

[1] W.X. Ma, J.-H. Lee, A transformed rational function method and exact solutions to the $3+1$ dimensional Jimbo-Miwa equation, Chaos Solitons Fractals.42 (2009) 1356-1363.

[2] J.P. Wu, A bilinear Bäcklund transformation and explicit solutions for a $(3+1)$-dimensional soliton equation, Chin. Phys. Lett. 25 (2008) 4192-4194.

[3] M.J. Ablowitz, H. Segur, Solitons and the Inverse Scattering Transform, SIAM, Philadelphia, 1981.

[4] M. Y. Adamu and E. Suleiman, On the Generalized Bilinear Differential Equations, IOSR Journal of Mathematics $3,(2013)$ (4) 24-30.
[5] W.X. Ma, T.W. Huang, Y. Zhang, A multiple exp-function method for nonlinear differential equations and its application, Phys. Scr. 82 (2010) 065003.

[6] R. Hirota (2004) The direct method in soliton theory. Cambridge, University, Press.

[7] W.X. Ma, A. Abdeljabbar, M.G. Asaad, Wronskian and Grammian solutions to a $(3+1)$-dimensional generalized KP equation, Appl. Math. Comput.217 (2011) 8722-8730.

[8] M. Y. Adamu and E. Suleiman On linear Superposition principle Applying to Hirota Bilinear Equations, American journal of computational and applied mathematics 3(1) (2013) 8-12.

[9] Magdy G. Asaad and W.X. Ma,Extended Gram-type Determinant, wave and rational solutions to $(3+1)$-dimensional nonlinear evolition equations,Appl. Math and Comp. 219 (2012), 213-225.

[10] F.C. You, T.C. Xia, D.Y. Chen, Decomposition of the generalized KP, cKP and mKP and their exact solutions, Phys. Lett. A 372 (2008) 3184-3194.

[11] A.M. Wazwaz, Multiple-soliton solutions for a $(3+1)$ dimensional generalized KP equation, Commun. Nonlinear Sci. Numer. Simul. 17 (2012) 491-495. 